Eur. Surg. Res. 1976;8:185-186

\title{
A Catheter for Vascular Surgery
}

\author{
S.-E. \\ Bergentz
}

Department of Surgery, Malmö General Hospital, Malmö

Prof. Sven-Erlk Bergentz, Professor of Surgery, Department of Surgery, Malmö General Hospital, University of Lund, Malmö (Sweden)

An intravascular catheter may be used in reconstructive vascular surgery for two different purposes. The first is to serve as a stant to facilitate the performance of the suture on the anastomosis. This is particularly useful in operations on small vessels and on veins. The second purpose is

Fig. 1. Helix catheter used in vascular surgery.

186

Bergentz

to have a shunt, thus avoiding the interruption of the blood flow through the vessel. This is of special importance in surgery of the vessels to the brain, and in surgery of the visceral vessels. For about 1 year we have a catheter available which fulfills both these tasks. It is made from a square teflon wire which is wound like a helix to form a catheter (fig. 1). Both the inner and outer surfaces of the catheter are smooth, and it is not possible to see that it actually is made up from a wire. In one end of the catheter, the teflon wire is drawn out as a free wire with an atraumatic needle in its end. This needle can be inserted through the vessel wall to bring out the wire. By pulling in this needle the whole catheter comes out as a wire. The catheter tolerates the blood pressure without leakage and is resistant to rough handling such as kinking and clamping. $\mathrm{T}$. The helix catheter has so far been used mainly in experimental surgery and small vessel surgery. It has a potential usefulness in other types of anastomoses such as in the common duct and urether where it can be left for a shorter or longer period of time. 